Discrete Comput Geom 30:311-320 (2003)

DOI: $10.1007 / \mathrm{s} 00454-003-0012-9$

\title{
Unavoidable Configurations in Complete Topological Graphs*
}

\author{
János Pach, ${ }^{1,2}$ József Solymosi, ${ }^{2,3}$ and Géza Tóth ${ }^{2}$ \\ ${ }^{1}$ Courant Institute, New York University, \\ New York, NY 10012, USA \\ pach@cims.nyu.edu \\ ${ }^{2}$ Rényi Institute, Hungarian Academy of Sciences, \\ Pf 127, H-1364 Budapest, Hungary \\ \{pach, solymosi, geza\}@ renyi.hu \\ ${ }^{3}$ Department of Mathematics, University of British Columbia, \\ Vancouver, British Columbia, Canada V6T 1Z4
}

\begin{abstract}
A topological graph is a graph drawn in the plane so that its vertices are represented by points, and its edges are represented by Jordan curves connecting the corresponding points, with the property that any two curves have at most one point in common. We define two canonical classes of topological complete graphs, and prove that every topological complete graph with $n$ vertices has a canonical subgraph of size at least $c \log ^{1 / 8} n$, which belongs to one of these classes. We also show that every complete topological graph with $n$ vertices has a non-crossing subgraph isomorphic to any fixed tree with at most $c \log ^{1 / 6} n$ vertices.
\end{abstract}

\section{Introduction, Results}

A topological graph $G$ is a graph drawn in the plane by Jordan curves, any two of which have at most one point in common. That is, it is defined as a pair $(V(G), E(G))$, where $V(G)$ is a set of points in the plane and $E(G)$ is a set of simple continuous arcs

\footnotetext{
* János Pach was supported by NSF Grant CCR-00-98246 and PSC-CUNY Research Award 644210034. József Solymosi was supported by the joint Berlin/Zürich graduate program Combinatorics, Geometry, Computation, financed by the German Science Foundation (DFG) and ETH Zürich. He is on leave from SZTAKI. Géza Tóth was supported by NSF Grant DMS-99-70071, OTKA-T-020914, and OTKA-F-22234.
} 


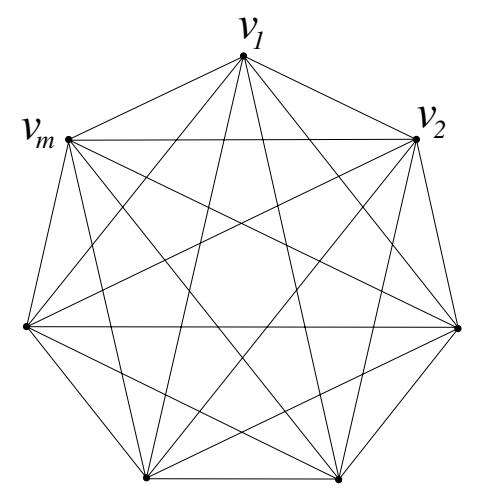

Fig. 1. The convex geometric graph $C_{m}$.

connecting them so that they satisfy the following conditions:

1. no arc passes through any other element of $V(G)$ different from its endpoints;

2. any two arcs have at most one point in common, which is either a common endpoint or a proper crossing.

$V(G)$ and $E(G)$ are the vertex set and edge set of $G$, respectively. We say that $H$ is a (topological) subgraph of $G$ if $V(H) \subseteq V(G)$ and $E(H) \subseteq E(G)$. Two topological graphs, $G$ and $H$, are called weakly isomorphic if there is an incidence preserving oneto-one correspondence between $(V(G), E(G))$ and $(V(H), E(H))$ such that two edges of $G$ intersect if and only if the corresponding edges of $H$ do (see [C]). If all edges of a topological graph are straight-line segments, then it is called a geometric graph. A geometric graph, whose vertices are in convex position, is called convex. Obviously, any two complete convex geometric graphs with $m$ vertices are weakly isomorphic to each other and to the convex geometric graph $C_{m}$, whose edge set consists of all sides and chords of a regular $m$-gon (see Fig. 1).

The fairly extensive literature on topological graphs focuses on very few special questions, and there is no standard terminology. For topological graphs, Erdős and Guy [EG] (see also [AR]) use the term "good drawings," while Gronau, Harborth, Mengersen, and Thürmann [GH], [HM1], [HM2], [HT] simply call them "drawings." For a complete topological graph, Ringel $[\mathrm{R}]$ and Mengersen $[\mathrm{M}]$ use the term "immersion." The most popular problems in this field are Turán's Brick Factory Problem [T] (Zarankiewicz's Conjecture [G] and other problems about crossing numbers, i.e., about the minimum number of crossings in certain drawings of a graph [PT2]) and Conway's Thrackle Conjecture [W], [LPS], [CN] (and other problems about the maximum number of crossings in certain drawings of a graph [HM3]).

The systematic study of geometric graphs was initiated by Erdős, Avital and Hanani [AH], Kupitz [K], and Perles. (See [P] and [PA, Chapter 14] for the most recent surveys on the subject.) It is not hard to see that every complete geometric graph $K_{n}$ of $n$ vertices has a non-crossing subgraph isomorphic to any triangulation of a cycle of length $n$ (see [GMPP]). Consequently, $K_{n}$ has a non-crossing subtree isomorphic to any fixed tree of $n$ vertices. In particular, $K_{n}$ has a non-crossing path of $n$ vertices and a non-crossing 
matching of size $\lfloor n / 2\rfloor$. On the other hand, it is known that $K_{n}$ has at least constant times $\sqrt{n}$ pairwise crossing edges [AEG]. Our aim is to establish analogous results for topological graphs.

Theorem 1.1. Every topological complete graph of n vertices has a non-crossing subgraph isomorphic to any fixed tree $T$ with at most $c \log ^{1 / 6} n$ vertices. In particular, it contains a non-crossing path with at least $c \log ^{1 / 6} n$ vertices.

According to a well-known theorem of Erdős and Szekeres [ES1], [ES2], any set of $n$ points in general position in the plane contains a subset with at least $c \log n$ elements which form the vertex set of a convex polygon. (Throughout this note, the letter $c$ appearing in different assertions denotes unrelated positive constants. The best known bound in the last statement is due Tóth and Valtr [TV].) The Erdôs-Szekeres Theorem can be reformulated, as follows.

Erdős-Szekeres Theorem. Every complete geometric graph with $n$ vertices has a complete geometric subgraph, weakly isomorphic to a convex complete graph $C_{m}$ with $m \geq c \log n$ vertices.

The situation is more complicated for topological graphs. In their study of topological complete graphs with $m$ vertices and with the maximum possible number, $\left(\begin{array}{c}m \\ 4\end{array}\right)$, of edge crossings, Harborth and Mengersen [HM3] found a drawing which contains no subgraph weakly isomorphic to $C_{5}$. We call this drawing, depicted in Fig. 2, twisted, and denote it by $T_{m}$.

We show that one cannot avoid both $C_{m}$ and $T_{m}$ in a sufficiently large complete topological graph.

Theorem 1.2. Every complete topological graph with $n$ vertices has a complete topological subgraph with $m \geq c \log ^{1 / 8} n$ vertices, which is weakly isomorphic either to a convex complete graph $C_{m}$ or to a twisted complete graph $T_{m}$.

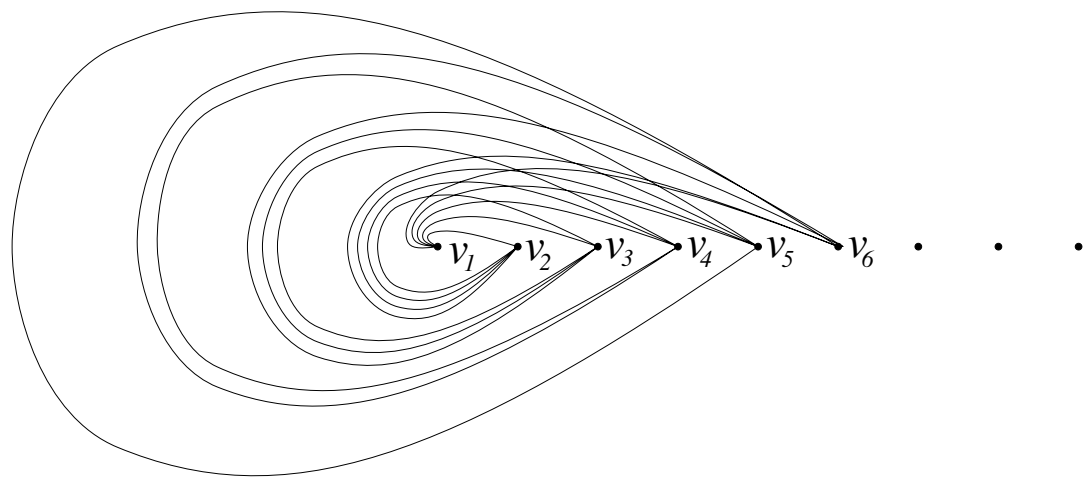

Fig. 2. The twisted drawing $T_{m}$. 
The arguments in the next section show that Theorem 2 easily implies a somewhat weaker version of Theorem 1 , where $\log ^{1 / 6} n$ is replaced by $\log ^{1 / 8} n$.

\section{Proofs}

Before we turn to the proofs, we rephrase the definitions of convex and twisted complete topological graphs.

Definition 2.1. Let $K_{m}$ be a complete topological graph on $m$ vertices. If there is an enumeration of the vertices, $\left\{u_{1}, u_{2}, \ldots, u_{m}\right\}$, such that

(i) two edges, $u_{i} u_{j}(i<j)$ and $u_{k} u_{l}(k<l)$, cross each other if and only if $i<k<j<l$ or $k<i<l<j$, then $K_{m}$ is called convex;

(ii) two edges, $u_{i} u_{j}(i<j)$ and $u_{k} u_{l}(k<l)$, cross each other if and only if $i<k<l<j$ or $k<i<j<l$, then $K_{m}$ is called twisted.

Let $K$ be a fixed complete topological graph with $n+1$ vertices. The edges of $K$ divide the plane into several cells, precisely one of which is unbounded. Without loss of generality, we can assume that there is a vertex $v_{0} \in V(K)$ on the boundary of the unbounded cell. Otherwise, we can apply a stereographic projection to transform $K$ into a drawing on a sphere, and then, by another projection, we can turn it into a topological graph weakly isomorphic to $K$, which satisfies the required property.

Consider all edges emanating from $v_{0}$, and denote their other endpoints by $v_{1}, v_{2}, \ldots$, $v_{n}$, in clockwise order. Color the triples $v_{i} v_{j} v_{k}, 1 \leq i<j<k \leq n$, with eight different colors, according to the following rules. Each color is represented by a zero-one sequence $a b c$ of length 3 . For any $i<j<k$,

1. set $a=0$ if the edges $v_{i} v_{j}, v_{0} v_{k} \in E(K)$ do not cross, and let $a=1$ otherwise;

2. set $b=0$ if the edges $v_{i} v_{k}, v_{0} v_{j} \in E(K)$ do not cross, and let $b=1$ otherwise;

3. set $c=0$ if the edges $v_{j} v_{k}, v_{0} v_{i} \in E(K)$ do not cross, and let $c=1$ otherwise.

It is easy to see that the complete topological subgraph of $K$ induced by the vertices $v_{0}, v_{i}, v_{j}, v_{k}$ (as any other complete topological graph with four vertices) has at most one pair of crossing edges. Therefore, we have

Claim 2.2. None of the colors $011,101,110$, or 111 can occur.

Proof of Theorem 1. Let $G$ be a topological complete graph on an $(n+1)$-element vertex set $V$. Use the same numbering, $v_{0}, v_{1}, \ldots, v_{n}$, of the vertices as in the previous section. For any $0<i<j$, we say that $v_{i}$ precedes $v_{j}$ (in notation, $v_{i} \prec v_{j}$ ). As before, color the triples $v_{i} v_{j} v_{k}(1 \leq i<j<k \leq n)$ with four colors, 000, 100, 010, and 001 .

Claim 2.3. There exists an $r$-element subset $U:=\left\{u_{1}, u_{2}, \ldots, u_{r}\right\} \subset\left\{v_{1}, v_{2}, \ldots, v_{n}\right\}$, $r \geq \sqrt{\log _{4}(n+1)}$, such that the triples $u_{i} u_{j} u_{k}$ and $u_{i} u_{j} u_{l}$ have the same color for any $i<j<k<l$. 
Proof. The construction is recursive. Let $U_{2}:=\left\{v_{1}, v_{2}\right\}$ and $V_{2}:=V \backslash\left\{v_{1}, v_{2}\right\}$. Suppose that, for some $2 \leq p<m$, we have already found two subsets $U_{p}=\left\{u_{1}, u_{2}, \ldots, u_{p}\right\}$ and $V_{p} \subset V$ with the properties

1. $u_{1} \prec u_{2} \prec \cdots \prec u_{p}$,

2. every element of $U_{p}$ precedes all elements of $V_{p}$,

3. $\left|V_{p}\right| \geq\left(\left|V_{p-1}\right|-1\right) / 4^{p}$.

Let $u_{p+1}$ be the smallest element of $V_{p}$ with respect to the ordering " $\prec$." Since we used four colors for coloring the triples, there is a subset $W \subset V_{p} \backslash\left\{u_{p+1}\right\}$ with $|W| \geq$ $\left(\left|V_{p}\right|-1\right) / 4^{p}$ such that, for each $1 \leq i \leq p$, all triples $u_{i} u_{p+1} w(w \in W)$ have the same color. Let $U_{p+1}:=U_{p} \cup\left\{u_{p+1}\right\}$ and $V_{p+1}:=W$. An easy computation shows that this procedure can be repeated at least $\left\lceil\sqrt{\log _{4}(n+1)}\right\rceil$ times.

Define the type of an edge $u_{i} u_{j}(i<j<m)$ as the color of a triple $u_{i} u_{j} u_{k}$ for any $k>j$. The type of $u_{i} u_{r}$ can be defined arbitrarily.

Let $G(100)$ and $G(001)$ denote the topological subgraphs of $G$ consisting of all edges of type 100 and 001 , respectively, whose both endpoints belong to $U=\left\{u_{1}, u_{2}, \ldots, u_{r}\right\}$. The topological subgraph consisting of all other edges of $G$ induced by $U$ (of types 000 and 010$)$ is denoted by $G^{\prime}$.

Claim 2.4. Let $i<j<k<m$.

(i) If $u_{i} u_{j}$ and $u_{j} u_{k}$ belong to $G(100)$, then so does $u_{i} u_{k}$.

(ii) If $u_{i} u_{j}$ and $u_{i} u_{k}$ belong to $G(001)$, then so does $u_{j} u_{k}$.

Proof. If $u_{i} u_{j}$ is of type 100, it must cross both $v_{0} u_{k}$ and $v_{0} u_{r}$. If the type of $u_{j} u_{k}$ is also 100 , it must cross $v_{0} u_{m}$, too. Using the assumption that two edges that share an endpoint cannot have any other point in common, we obtain that $u_{i} u_{k}$ must cross $v_{0} u_{m}$, which implies that its type is also 100 (see Fig. 3). This proves part (i). Part (ii) can be established similarly.

Claim 2.5. If $G(100), G(001)$, or $G^{\prime}$ contains a complete subgraph of size $m:=$ $\left\lceil r^{1 / 3}\right\rceil$, then $G$ has a non-crossing subgraph isomorphic to any tree of $m$ vertices.

Proof. Suppose that $w_{1} \prec w_{2} \prec \cdots \prec w_{m}$ induce a complete (topological) subgraph in $G(100)$. It is easy to see that this subgraph is twisted, i.e., it is weakly isomorphic to $T_{m}$. Take an arbitrary tree $T$ with $m$ vertices. Starting at any vertex $z_{1} \in V(T)$, explore all other vertices of $T$ using breadth-first search. Let $z_{1}, z_{2}, \ldots, z_{m}$ be a numbering of the elements of $V(T)$, in the order in which they are encountered by the algorithm. Then the embedding $f\left(z_{i}\right)=w_{i}(1 \leq i \leq r)$ maps $T$ into a non-crossing copy of $T$ in $G(100)$, and we are done.

The case when $G(001)$ contains a complete subgraph of size $m$ can be treated similarly.

Assume now that $G^{\prime}$ has a complete subgraph with $m$ vertices, $w_{1} \prec w_{2} \prec \cdots \prec w_{r}$. It is easy to see that if two edges, $w_{i} w_{j}(i<j)$ and $w_{k} w_{l}(k<l)$, cross each other, then 

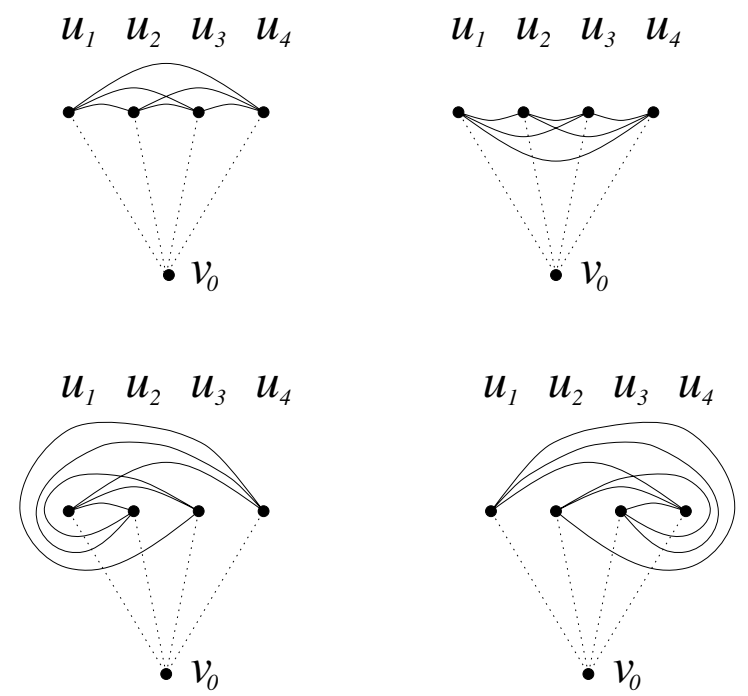

Fig. 3. All triples are of type 000, 010, 001, and 100, respectively.

we have $i<k<j<l$ or $k<i<l<j$. In other words, if two edges of this subgraph cross each other, the corresponding edges also cross in a drawing on the same vertex set, weakly isomorphic to the convex drawing $C_{m}$. Clearly, $C_{m}$ contains a non-crossing copy of every tree with $m$ vertices, so the same is true for $G^{\prime}$.

In view of the last claim, it remains to prove that at least one of $G(100), G(001)$, and $G^{\prime}$ has a complete subgraph of size $m=\left\lceil r^{1 / 3}\right\rceil$. Suppose, in order to obtain a contradiction, that this is not the case.

If some element $u \in U=\left\{u_{1}, u_{2}, \ldots, u_{r}\right\}$ had at least $m-1$ larger neighbors in $G(001)$ with respect to the ordering $\prec$, then, by Claim 2.4(ii), these neighbors together with $u$ would induce a complete subgraph in $G(001)$, a contradiction.

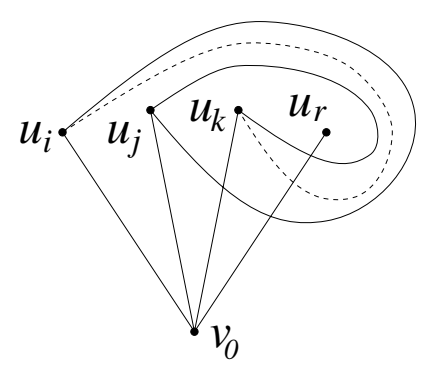

Fig. 4. $u_{i} u_{k}$ must cross $v_{0} u_{r}$. 
Now we recursively construct a sequence $w_{1} \prec w_{2} \prec \cdots$ consisting of at least $r^{2 / 3}$ elements of $U$, which form an independent set in $G(001)$ (i.e., they induce a complete subgraph in $\left.G(100) \cup G^{\prime}\right)$.

Let $W_{0}:=\emptyset$ and $U_{0}:=\left\{u_{1}, u_{2}, \ldots, u_{r}\right\}$. Suppose that, for some $p<m^{2 / 3}$, we have already found two subsets $W_{p}=\left\{w_{1}, w_{2}, \ldots, w_{p}\right\}$ and $U_{p} \subset\left\{u_{1}, u_{2}, \ldots, u_{r}\right\}$, such that

1. $W_{p}$ is an independent set in $G(001)$,

2. every element of $W_{p}$ precedes every element of $U_{p}$,

3. there is no edge between $W_{p}$ and $U_{p}$,

4. $\left|U_{p}\right| \geq r-p(m-1)$.

If $U_{p} \neq \emptyset$, let $w_{p+1}$ be the smallest element of $U_{p}$ with respect to the ordering $\prec$, and set $W_{p+1}:=W_{p} \cup\left\{w_{p+1}\right\}$. Let $U_{p+1}$ denote the set obtained from $U_{p}$ by the deletion of $w_{p+1}$ and its larger neighbors. Clearly, we have $\left|U_{p+1}\right| \geq\left|U_{p}\right|-m+1$, so that this procedure can be repeated at least $\left\lceil r^{2 / 3}\right\rceil$ times.

Define the rank of any element $w \in W:=\left\{w_{1}, w_{2}, \ldots\right\}$, as the number of vertices of the longest monotone path (with respect to $\prec$ ) which ends at $w$ in the subgraph of $G(100)$ induced by $W$. There is no element whose rank is at least $r^{1 / 3}$, otherwise, by Claim 2.4(i), the vertices of the corresponding path would induce a complete subgraph of size at least $m$ in $G(100)$, contradicting our assumptions.

Therefore, we can suppose that at least $r^{1 / 3}$ elements of $W$ have the same rank. According to the definitions, these elements form an independent set in $G(100)$ as well as in $G(001)$. Thus, they induce a complete subgraph in $G^{\prime}$, again a contradiction. This proves Theorem 1 .

Proof of Theorem 2. Let $m=\left\lceil r^{1 / 4}\right\rceil$ now. Just like in the proof of Theorem 1, one can show that either one of $G(100)$ and $G(001)$ has a complete subgraph of size $m$, or $G^{\prime}$ has a complete subgraph of size $s=\left\lceil r^{1 / 2}\right\rceil$. In cases when $G(100)$ or $G(001)$ has a complete subgraph of size $m$, we are done because that subgraphs are twisted (see Claim 2.2). Assume now that $G^{\prime}$ has a complete subgraph $G^{\prime \prime}$ of size $s$.

Define the rank of any vertex $w$ of $G^{\prime \prime}$ as the length of the longest path $w_{1} \prec w_{2} \prec$ $\cdots \prec w_{k}=w$ in $G^{\prime \prime}$ with all edges $w_{i} w_{i+1}$ of type 000 .

Suppose first that all vertices have rank less than $m$. Since $m \geq \sqrt{s}$, there are $m$ vertices of $G^{\prime \prime}$ of the same rank, so all edges among them are of type 010 , therefore they induce a complete convex topological graph of $m$ vertices.

So, we can assume that there is a vertex of rank $m$, that is, there are vertices of $G^{\prime \prime}$ $w_{1} \prec w_{2} \prec \cdots \prec w_{m}$ with all edges $w_{i} w_{i+1}$ of type 000 .

Suppose that there is a triple $z_{i} \prec z_{j} \prec z_{k} \subset w_{1} \prec w_{2} \prec \cdots \prec w_{m}$ of color 010. Then $z_{i} z_{j}$ is of type 010 . Choose a closest pair $z_{i} \prec z_{j}$ of type 010 on the path $w_{1} \prec w_{2} \prec \cdots \prec w_{m}$. The vertex next to $z_{i}$ in the path is denoted by $z_{i}^{+}$. Clearly, $z_{i}^{+} \neq z_{j}$ and $z_{i}^{+} v_{0}$ has no crossing with the edge $z_{i} z_{k}$, because $z_{i} z_{i}^{+}$has type 000. However, then $z_{i}^{+} \prec z_{j} \prec z_{k}$ should have color 010, too, so $z_{i}^{+} z_{j}$ is also of type 010, contradicting the "minimality" of $z_{i} \prec z_{j}$ (see Fig. 5). Consequently, all edges among $w_{1} \prec w_{2} \prec \cdots \prec w_{m}$ are of type 000, therefore they induce a complete convex topological graph of $m$ vertices. 


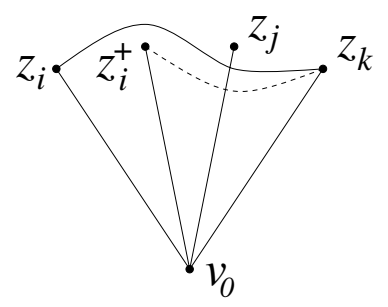

Fig. 5. $z_{i}^{+} z_{j}$ is also of type 010 .

\section{Concluding Remarks}

I. The following statement is a direct corollary of the first result in [PSS].

Theorem 3.1. Every complete topological graph of $n$ vertices contains at least $c \log n / \log \log n$ pairwise crossing edges.

II. Both $C_{m}$ and $T_{m}$, the convex and the twisted topological graphs with $m$ vertices, respectively, determine precisely $\left(\begin{array}{c}m \\ 4\end{array}\right)$ edge crossings. Therefore, the following theorem of Harborth et al. [HMS] is an immediate consequence of Theorem 2.

Corollary 3.2. For any positive integer $m$, there exists a smallest number $n(m)$ such that every complete topological graph with at least $n(m)$ vertices has a complete subgraph with $m$ vertices and with $\left(\begin{array}{c}m \\ 4\end{array}\right)$ crossings between its edges.

In fact, for large values of $m$, Theorem 2 implies a better bound on the function $n(m)$ than the proof given in [HMS].

III. Let $F$ denote the graph obtained from a complete graph of five vertices by subdividing each of its edges with an extra vertex. Given a complete topological graph $K_{n}$ of $n$ vertices, define an abstract graph $G$. Let the vertex set of $G$ consist of $\lfloor n / 2\rfloor$ edges of $K_{n}$, no two of which share an endpoint. Let two vertices, $e, e^{\prime} \in E\left(K_{n}\right)$, be joined by an edge of $G$ if and only if $e$ and $e^{\prime}$ cross each other. It is easy to see that $G$ does not contain $F$ as an induced subgraph (see, e.g., [EET]).

It follows from a theorem of Erdôs and Hajnal [EH] that, if a graph with $m$ vertices does not contain some fixed induced subgraph $F$, then it must have either an empty or a complete subgraph with at least $e^{c \sqrt{\log m}}$ vertices, where $c>0$ is a constant depending on $F$. Putting these two facts together, we obtain

Corollary 3.3. Any topological complete graph with $n$ vertices has at least $e^{c \sqrt{\log n}}$ edges that are either pairwise disjoint or pairwise crossing.

This suggests that the bounds in Theorems 1 and 3.2 are far from being optimal. We conjecture that both estimates can be replaced by $n^{\delta}$, for some $\delta>0$. As was pointed out in the Introduction, this holds for geometric graphs. 
IV. In the case of geometric graphs, one can introduce several partial orderings on the set of edges (see [PT1] and [PA]). This allows us to apply Dilworth's theorem in place of Ramsey's theorem, to find much larger homogeneous substructures.

\section{References}

[AH] S. Avital and H. Hanani: Graphs (in Hebrew), Gilyonot Lematematika 3 (1966), 2-8.

[AR] D. Archdeacon and B. R. Richter: On the parity of crossing numbers, J. Graph Theory 12 (1988), 307-310.

[AEG] B. Aronov, P. Erdős, W. Goddard, D. Kleitman, M. Klugerman, J. Pach, and L. Schulman: Crossing families, Combinatorica 14 (1994), 127-134.

[C] Ch. J. Colbourn: On drawings of complete graphs, J. Combin. Inform. System Sci. 6 (1981), 169-172.

[CN] G. Cairns and Y. Nikolayevsky: Bounds for generalized thrackles, Discrete Comput. Geom. 23 (2000), 191-206.

[EET] G. Ehrlich, S. Even, and R. E. Tarjan: Intersection graphs of curves in the plane, J. Combin. Theory Ser. B 21 (1976), 8-20.

[EG] P. Erdôs and R. K. Guy: Crossing number problems, Amer. Math. Monthly 80 (1973), 52-58.

[EH] P. Erdős and A. Hajnal: Ramsey-type theorems, Discrete Appl. Math. 25 (1989), 37-52.

[ES1] P. Erdős and G. Szekeres: A combinatorial problem in geometry, Compositio Math. 2 (1935), 463470.

[ES2] P. Erdôs and G. Szekeres: On some extremum problems in elementary geometry, Ann. Univ. Sci. Budapest. Eötvös Sect. Math. III-IV (1960-61), 53-62.

[G] R. K. Guy: The decline and fall of Zarankiewicz's theorem, in: Proof Techniques in Graph Theory, Academic Press, New York, 1969, pp. 63-69.

[GH] H.-D. Gronau and H. Harborth: Numbers of nonisomorphic drawings for small graphs, in: Proceedings of the Twentieth Southeastern Conference on Combinatorics, Graph Theory, and Computing (Boca Raton, FL, 1989), Congressus Numerantium 71, Utilitas Math., Winnipeg, 1990, pp. 105114.

[GJ] M. R. Garey and D. S. Johnson: Crossing number is NP-complete, SIAM J. Algebraic Discrete Methods 4 (1983), 312-316.

[GMPP] P. Gritzmann, B. Mohar, J. Pach, and R. Pollack: Embedding a planar triangulation with vertices at specified points, Amer. Math. Monthly 98 (1991), 165-166.

[HM1] H. Harborth and I. Mengersen: Edges without crossings in drawings of complete graphs, J. Combin. Theory Ser. B 17 (1974), 299-311.

[HM2] H. Harborth and I. Mengersen: Edges with at most one crossing in drawings of the complete graph, in: Topics in Combinatorics and Graph Theory (Oberwolfach, 1990), Physica Verlag, Heidelberg, 1990, pp. 757-763.

[HM3] H. Harborth and I. Mengersen: Drawings of the complete graph with maximum number of crossings, in: Proceedings of the Twenty-Third Southeastern International Conference on Combinatorics, Graph Theory, and Computing (Boca Raton, FL, 1992), Congressus Numerantium 88, Utilitas Math., Winnipeg, 1992, pp. 225-228.

[HMS] H. Harborth, I. Mengersen, and R. H. Schelp: The drawing Ramsey number $\operatorname{Dr}\left(K_{n}\right)$, Austral. $J$. Combin. 11 (1995), 151-156.

[HT] H. Harborth and Ch. Thürmann: Minimum number of edges with at most $s$ crossings in drawings of the complete graph, in: Proceedings of the Twenty-Fifth Southeastern International Conference on Combinatorics, Graph Theory and Computing (Boca Raton, FL, 1994), Congressus Numerantium 102, Utilitas Math., Winnipeg, 1994, pp. 83-90.

[K] Y. S. Kupitz: Extremal Problems in Combinatorial Geometry, Lecture Notes Series 53, Aarhus Universitet, Matematisk Institut, Aarhus, 1979.

[LPS] L. Lovász, J. Pach, and M. Szegedy: On Conway's thrackle conjecture, Discrete Comput. Geom. 18 (1997), 369-376.

[M] I. Mengersen: Die Maximalzahl von kreuzungsfreien Kanten in Darstellungen von vollständigen n-geteilten Graphen (in German), Math. Nachr. 85 (1978), 131-139. 
[P] J. Pach: Geometric graph theory, in: Surveys in Combinatorics, 1999 (Canterbury), London Mathematical Society Lecture Note Series 267, Cambridge University Press, Cambridge, 1999, pp. 167200.

[PA] J. Pach and P. K. Agarwal: Combinatorial Geometry, Wiley-Interscience, New York, 1995.

[PSS] J. Pach, F. Shahrokhi, and M. Szegedy: Applications of crossing numbers, Algorithmica 16 (1996), 111-117.

[PT1] J. Pach and J. Törôcsik: Some geometric applications of Dilworth's theorem, Discrete Comput. Geom. 12 (1994), 1-7.

[PT2] J. Pach and G. Tóth: Which crossing number is it, anyway? J. Combin. Theory Ser. B 80 (2000), 225-246.

[R] G. Ringel: Extremal problems in the theory of graphs, in: Theory of Graphs and Its Applications (Proc. Sympos. Smolenice, 1963), Publ. House Czechoslovak Acad. Sci., Prague, 1964, pp. 85-90.

[T] P. Turán: A note of welcome, J. Graph Theory 1 (1977), 7-9.

[TV] G. Tóth and P. Valtr: Note on the Erdős-Szekeres theorem, Discrete Comput. Geom. 19 (1998), 457-459.

[W] D. R. Woodall: Thrackles and deadlock, in: Combinatorial Mathematics and Its Applications (Proc. Conf., Oxford, 1969), Academic Press, London, 1971, pp. 335-347.

[WB] A. T. White and L. W. Beineke: Topological graph theory, in: Selected Topics in Graph Theory (L. W. Beineke and R. J. Wilson., eds.), Academic Press, [Harcourt Brace Jovanovich], London [New York], 1983, pp. 15-49.

Received July 21, 2000, and in revised form January 23, 2001. Online publication July 14, 2003. 